
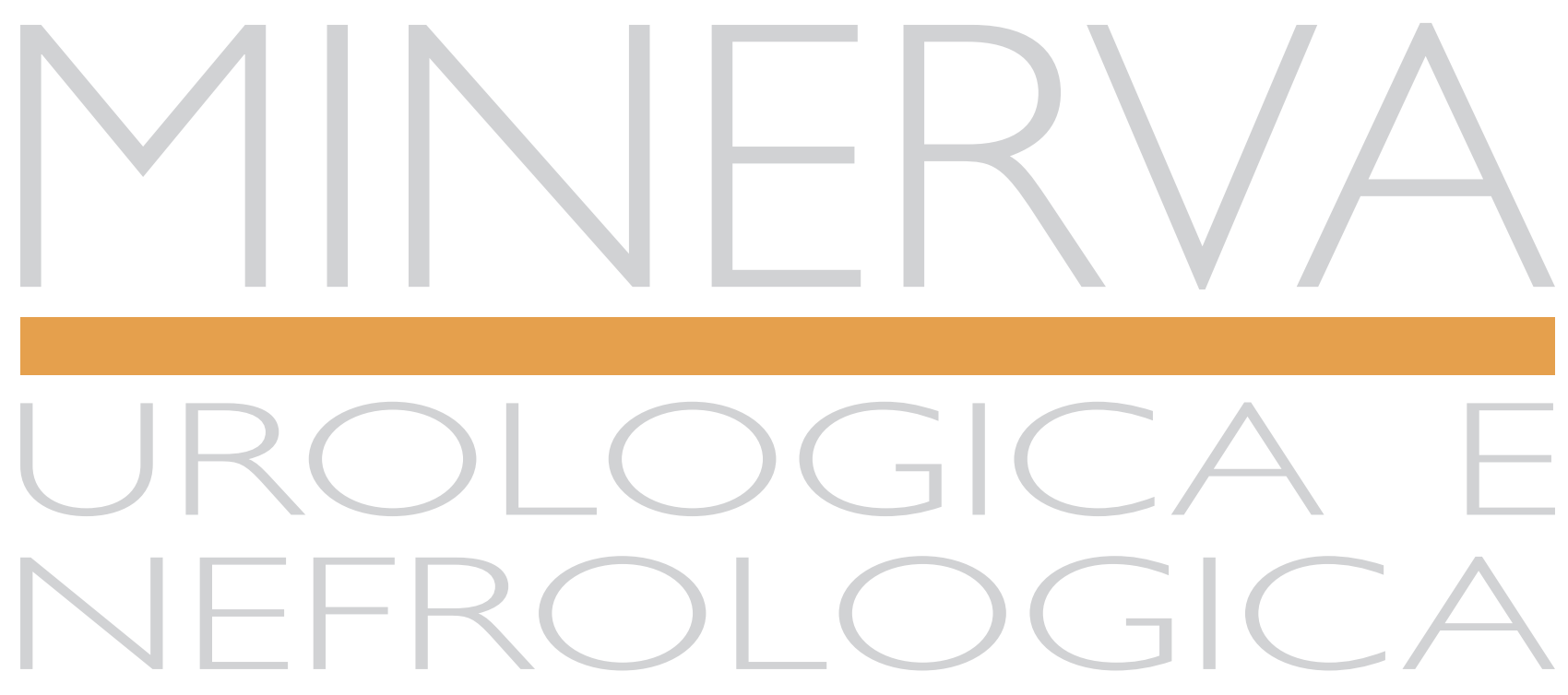

$V O L U M E 70 \cdot N O \cdot I \cdot F E B R \cup A R Y 2018$
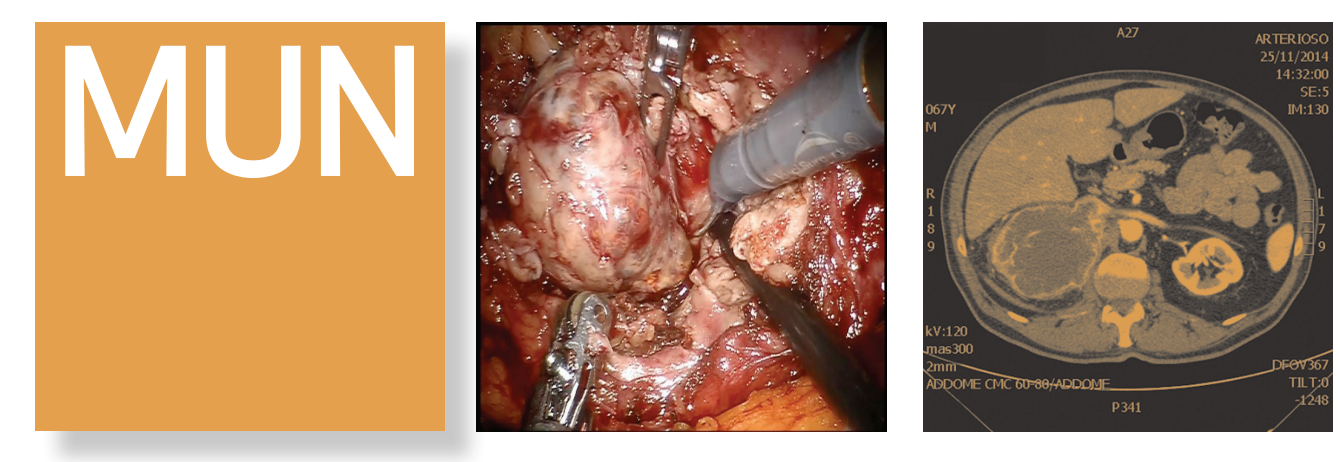

Minerva

Urology and

Nephrology
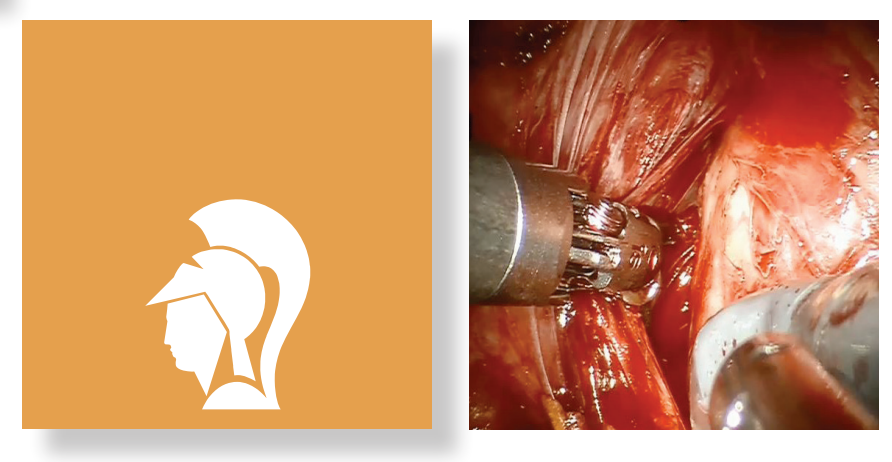

$E D|Z| O N|\cdot M| N E R \vee A \cdot M E D \mid C A$ 


\title{
RECORd1 project: what have we learned?
}

\author{
Andrea MINERVINI $1^{*}$, Andrea MARI 1, Vincenzo FICARRA 2, \\ Francesco PORPIGLIA ${ }^{3}$, Marco CARINI ${ }^{1}$
}

\begin{abstract}
1Department of Urology, University of Florence, Careggi Hospital, Florence, Italy; ${ }^{2}$ Gaetano Barresi Department of Human and Pediatric Pathology, Urologic Section, University of Messina, Messina, Italy; ${ }^{3}$ Department of Urology, University of Turin, San Luigi Gonzaga Hospital, Orbassano, Turin, Italy

*Corresponding author: Andrea Minervini, Department of Urology, University of Florence, Careggi Hospital, San Luca Nuovo, Florence, Italy. E-mail: andrea.minervini@unifi.it
\end{abstract}

T he Italian Registry of Conservative Renal Surgery (RECORd1 Project) is a multicenter, observational, prospective project developed in 2008 promoted by the Leading Urological Non-Profit Foundation for Advanced Research (LUNA) of the Italian Society of Urology (SIU) involving 19 Italian centers. The aim of this project was to offer a comprehensive view of the national trend of utilization of conservative kidney surgery and to offer a "real-life" description of the clinical, perioperative, pathological as well as oncological and functional results in patients treated with NSS for renal tumors in Italy from 2009 to 2012, to provide evidence on a significant gap of knowledge, such as: 1) the impact of the surgical approach (OPN vs. LPN vs. RAPN) on outcomes according to tumors' and patients' characteristics; 2) the feasibility of widening the indications of minimally invasive techniques to larger and more complex tumors; 3) the performance of different surgical techniques (enucleation vs. enucleoresection/ standard partial nephrectomy); 4) the trend in the use of hemostatic agents and their putative protective effect against bleeding and finally; 5) the impact of several epidemiological and clinical factors on postoperative outcomes (complications, renal function $[\mathrm{RF}]$ and oncologic outcome) after NSS.

This was the first multicenter online web- based database for kidney surgery in Italy and it comprises 6 main categories: 1) anthropometric and preoperative data; 2) imaging, indications and comorbidities; 3 ) intraoperative data; 4) postoperative data; 5) histopathological analysis; 6) follow-up. All data were centrally recorded on a data server.

Overall, the clinical data of 1055 patients were recorded. Patients undergoing NSS were found to be increasingly younger, healthier, and with larger tumor diameter over the period considered. Notably, a constant decrease in the use of the OPN both for cT1a and cT1b over time was reported. RAPN increased in the last two years, while LPN seemed a wellestablished and adopted procedure in the Italian centers resulting in a slight decrease of its utilization over time. Enucleoresection was the most widely adopted technique (60\%) among centers with a not negligible use of enucleation $(40 \%)$. The attitude towards an off-clamp procedure increased over time for all the three approaches. The WIT did not differ significantly, and the median WIT was always $<20$ minutes. ${ }^{1}$

LPN was confirmed to be not inferior to OPN in clinical T1a tumors in a matched pair analysis of patients within the RECORd1 project. Indeed, no significant difference in achieving the TRIFECTA outcome and in renal function decrease at the $6^{\text {th }}$ month from surgery was reported after OPN and LPN al- 
though the latter was associated with a significantly longer WIT. ${ }^{2}$ In another study within the RECORd 1 project, patients who underwent RAPN showed equivalent surgical, early oncological and functional outcomes and a significantly lower overall postoperative complication rate compared to OPN. ${ }^{3}$ This result was confirmed also for the treatment of cT1b tumors. The study showed that clinical $\mathrm{T} 1 \mathrm{~b}$ renal tumors could be safely treated by a minimally invasive approach (either LPN or RAPN) in high-volume centres, and that RAPN was associated with a significantly lower WIT and blood loss compared with LPN.4, 5

The analysis on the performance of different surgical techniques (enucleation vs. enucleoresection/standard partial nephrectomy) showed that simple enucleation (SE) is associated with a significantly lower blood loss and shorter operative time, but comparable medical and surgical complications to standard PN. Furthermore, standard PN was an independent predictor of positive surgical margins (PSM) with a 4.7-fold increased risk compared to SE. ${ }^{6}$ Another study, evaluating the trend in the use of hemostatic agents and their putative protective effect against bleeding showed that hemostatic agents are widely adopted during NSS, $83 \%$ of the procedures required the use of an hemostatic agent, but no differences were found in terms of postoperative outcomes between TachoSil ${ }^{\circledR}$ (Takeda Pharma), Floseal ${ }^{\circledR}$ (Takeda, Inc.) and their non-use (TriMatch statistical analysis after balancing for confounding factors) thus suggesting that adding hemostatic agents to renorrhaphy during NSS does not provide better surgical outcomes. ${ }^{7}$

In the RECORd1 project, prognostic factors of postoperative surgical complications, oncological and functional outcomes were also investigated. The presence of comorbidities and the surgical open (compared to robotic) approach were independent predictors of overall and major surgical complications. ${ }^{8}$ Conversely, age, body mass index (BMI), nephrometry parameters and surgical indication were not. ${ }^{8}$

From an oncological perspective, higher age, upper pole tumor location, enucleoresection versus enucleation, and Fuhrman 3-4 nuclear grade were found to be independent predictors of PSM. ${ }^{9}$ Imperative indication and PSM status were independent predictors of ipsilateral renal recurrence. ${ }^{10}$

The surgical modifiable factors (the open and laparoscopic vs robotic approach and the on-clamp vs off-clamp) were independent predictors of immediate ( $3^{\text {rd }}$ postoperative day) and early ( $1^{\text {st }}$ month) functional deterioration after PN, conversely other clinical non-modifiable factors (female gender, age and baseline renal function) were correlated to immediate and early renal function decline but also to late (24th month) functional deterioration. ${ }^{11}$ Uncontrolled diabetes mellitus was independently correlated to late functional impairment, only. The late functional impairment together with BMI were showed to be independent predictors of cardiovascular events at a median follow-up of 86 months.

The excellent results of this study have prompted the scientific committee to promote a new study on renal surgery (including radical and ablative therapies) for renal tumors: the RECORd2 project prospectively included over 4300 patients undergone surgery from 2013 to 2016 in 34 Italian centers. Follow-up of these patients is ongoing as well as statistical analyses. This project focused on the indication and the effectiveness of the currently available therapeutic strategies for the treatment of patients with localized renal tumors. The results of RECORd2 project will soon be published.

While the RECORd 2 project is ending (inclusion phase closed in December 2016 and follow-up recording will close December 2018), the i-RECORD (international) project is about to start early 2018 . The project will reproduce the design of a prospective, observational, adaptable, clinical trial, recalling the concept of multi-arm multistage platform. The aim of the study is to define a-priori groups of patients selected with renal neoplasia based on tumors' and patients' characteristics and the hospital resources. Designing a subdivision into parallel and dynamic study arms might provide the base to set research questions of current and future interest in the treatment of localized/locally advanced renal tumors. 


\section{References}

1. Schiavina R, Mari A, Antonelli A, Bertolo R, Bianchi G, Borghesi M, et al. A snapshot of nephron-sparing surgery in Italy: A prospective, multicenter report on clinical and perioperative outcomes (the RECORd 1 project). Eur J Surg Oncol 2015;41:346-52.

2. Minervini A, Siena G, Antonelli A, Bianchi G, Bocciardi AM, Cosciani Cunico S, et al. Open versus laparoscopic partial nephrectomy for clinical T1a renal masses: A matched-pair comparison of 280 patients with TRIFECTA outcomes (RECORd Project). World J Urol 2014;32:257-63.

3. Ficarra V, Minervini A, Antonelli A, Bhayani S, Guazzoni G, Longo N, et al. A multicentre matched-pair analysis comparing robot-assisted versus open partial nephrectomy. BJU Int 2014;113:936-941.

4. Porpiglia F, Mari A, Bertolo R, Antonelli A, Bianchi G, Fidanza F, et al. Partial Nephrectomy in Clinical T1b Renal Tumors: Multicenter Comparative Study of Open, Laparoscopic and Robot-assisted Approach (the RECORd Project). Urology 2016;89:45-51.

5. Minervini A, Mari A, Carini M, Porpiglia F. Author Reply. Urology 2016;89:52-53.

6. Longo N, Minervini A, Antonelli A, Bianchi G, Bocciardi AM, Cosciani Cunico S, et al. Simple enucleation versus standard partial nephrectomy for clinical T1 renal masses: Perioperative outcomes based on a matched-pair comparison of 396 patients (RECORd project). Eur J Surg Oncol 2014;40:762-8.
7. Antonelli A, Minervini A, Mari A, Bertolo R, Bianchi G, Lapini A, et al. TriMatch comparison of the efficacy of FloSeal versus TachoSil versus no hemostatic agents for partial nephrectomy: Results from a large multicenter dataset. Int J Urol 2015;22:47-52.

8. Mari A, Antonelli A, Bertolo R, Bianchi G, Borghesi $\mathrm{M}$, Ficarra V, et al. Predictive factors of overall and major postoperative complications after partial nephrectomy: Results from a multicenter prospective study (The RECORd 1 project). Eur J Surg Oncol 2017;43:823-30.

9. Schiavina R, Serni S, Mari A, Antonelli A, Bertolo R, Bianchi $\mathrm{G}$, et al. A prospective, multicenter evaluation of predictive factors for positive surgical margins after nephron-sparing surgery for renal cell carcinoma: The RECORd1 Italian project. Clin Genitourin Cancer 2015;13:165-70.

10. Minervini A, Mari A, Campi R, Novara G, Antonelli A, Bertolo R, et al. Predictors of local recurrence after partial nephrectomy: Results from two-years follow up of a prospective multicentre study (RECORd 1 project). Eur Urol Suppl 2017;16:e1370-1

11. Antonelli A, Mari A, Longo N, Novara G, Porpiglia F, Schiavina R, et al. Role of Clinical and Surgical Factors for the Prediction of Immediate, Early and Late Functional Result and its Relatonship with Cardivascular Outcome After Partial Nephrectomy: Results from the Prospective Multicenter RECORd 1 Project. J Urol 2017 Nov 14. [Epub ahead of print]

Contributors -Daniele Amparore, Alessandro Antonelli, Walter Artibani, Riccardo Bertolo, Giampaolo Bianchi, Aldo Massimo Bocciardi, Marco Borghesi, Eugenio Brunocilla, Riccardo Campi, Andrea Chindemi, Mario Falsaperla, Cristian Fiori, Maria Furlan, Fernando Fusco, Saverio Giancane, Vincenzo Li Marzi, Nicola Longo, Vincenzo Mirone, Giuseppe Morgia, Giacomo Novara, Bernardo Rocco, Bruno Rovereto, Riccardo Schiavina, Sergio Serni, Claudio Simeone, Riccardo Tellini, Carlo Terrone, Paolo Verze, Alessandro Volpe, Filiberto Zattoni.

Conflicts of interest.-The authors certify that there is no conflict of interest with any financial organization regarding the material discussed in the manuscript.

Manuscript accepted: November 27, 2017. - Manuscript received: November 23, 2017.

(Cite this article as: Minervini A, Mari A, Ficarra V, Porpiglia F, Carini M. RECORd1 project: what have we learned? Minerva Urol Nefrol 2018;70:1-3. DOI: 10.23736/S0393-2249.17.03099-5) 